\title{
Recurrent intracranial meningioma with multiple pulmonary metastases: A case report
}

\author{
KE-DA WANG ${ }^{1}$, YI-BING SU ${ }^{1}$ and YAN ZHANG ${ }^{2}$ \\ ${ }^{1}$ Department of Neurosurgery, Beijing Jishuitan Hospital, Beijing 100035; ${ }^{2}$ Department of Neurosurgery, \\ Beijing Tiantan Hospital, Capital Medical University, Beijing 100050, P.R. China
}

Received September 8, 2014; Accepted August 5, 2015

DOI: $10.3892 / \mathrm{ol} .2015 .3670$

\begin{abstract}
Meningiomas are slow-growing tumors, which are generally considered to be benign and rarely metastasize. Although cases of extracranial metastatic meningioma have previously been reported, multiple pulmonary metastases from a benign intracranial meningioma is particularly rare. In the present report, a case of recurrent transitional meningioma with multiple lung nodules, which were demonstrated to be metastatic meningioma, is presented. A 54-year-old female patient received surgical resection of the tumor located in the left base of the middle cranial fossa in 2006. Post-surgery pathological examination indicated a transitional meningioma of World Health Organization grade I. The tumor recurred at the original site 1 year and 3 months later and was completely surgically removed once again. Radiotherapy was administered following the second surgery. Gamma Knife was used to remove the recurrent tumor 18 months following the second surgery. Simultaneously, a chest computed tomography scan revealed multiple pulmonary nodules, which were demonstrated to be metastatic meningioma following wedge resection of the superior lobe of the right lung. The clinical behavior of meningioma does not always correlate with the classification of meningioma. A higher rate of cellular proliferation is not essential for extracranial metastasis, and an individual meningioma of any type may metastasize. Comprehensive examinations should be performed for patients with a history of recurrent intracranial meningioma to detect any distant metastases as early as possible, even when the primary tumor is benign.
\end{abstract}

\section{Introduction}

Meningiomas are typically benign intracranial tumors, and rarely metastasize extracranially (1). Metastasis occurs in $<1 \%$ of meningiomas, and common metastatic sites include

Correspondence to: Dr Yan Zhang, Department of Neurosurgery, Beijing Tiantan Hospital, Capital Medical University, 6 Tiantan Xili, Beijing 100050, P.R. China

E-mail: yanzhang135@163.com

Key words: meningioma, metastasis, lung the lung, liver, lymph node, bone and pleura (2). Tumors with malignant histological features are associated with a higher metastatic rate (3); however, there are reports of metastasis from benign meningiomas $(2,4)$. Rawat et al $(5)$ reported 77 cases of meningioma with extracranial metastasis in 1995 , and $\sim 70$ additional cases of meningioma with extracranial metastasis were identified in the literature (2-4,6-27). There are no definitive criteria for the prediction of metastasis in meningiomas, and the discovery of metastasis frequently occurs following recurrence of the primary tumor (23-25). Therefore comprehensive examinations for patients with a history of recurrent intracranial meningioma are required for the detection of distant metastases, despite the tumor being benign.

\section{Case report}

A 54-year-old female was admitted to the Second Hospital of Hebei Medical University (Shijiazhuang, China) with primary symptoms of a progressive headache for the preceding 3 months, and nausea accompanied by vomiting for 4 days. Physical examination was normal, except for bilateral papilloedema. Chest plain radiograph was normal. Magnetic resonance imaging (MRI) revealed a contrast-enhancing tumor in the left base of the middle cranial fossa.

A gross total resection of the tumor $(\sim 6 \times 5 \times 5 \mathrm{~cm})$ was performed via a left fronto-temporal craniotomy (Simpson Grade III) (28). Post-surgical pathological examination revealed a transitional meningioma with meningiothelial and fibroblastic components, which was classified as World Health Organization grade I (29). Immunohistochemical examination was not performed to explore the histological features. Postoperative computed tomography (CT) indicated that the tumor had been completely removed.

One year and 3 months later, the patient experienced tinnitus in the left ear and dizziness for 1 month. Magnetic resonance imaging (MRI) revealed a recurrent mass of $4 \times 5 \times 5 \mathrm{~cm}$ at the original site and the tumor was once again completely resected (Fig. 1). Pathological diagnosis was transitional meningioma with similar characteristics to those of the original tumor (Fig. 2). Following surgery, significant signs of impairment to the oculomotor nerve were observed. Radiotherapy was administered (specific radiation dose unknown) 1 month following the second surgery. 

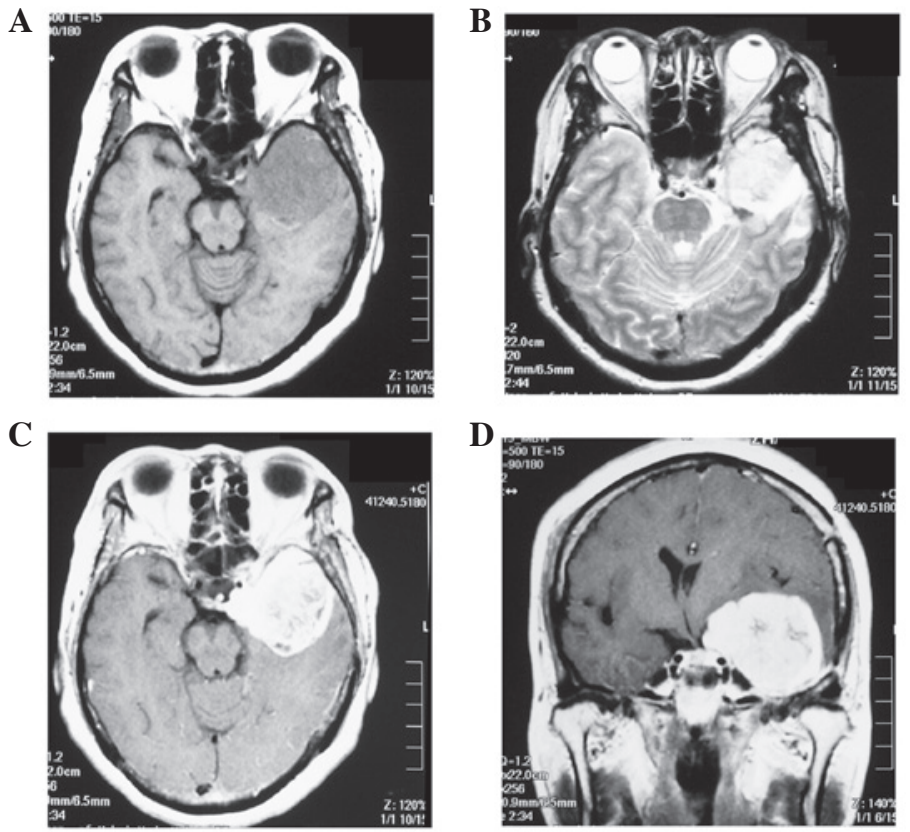

Figure 1. (A) MRI axial T1WI, (B) MRI axial T2WI, (C and D) axial and coronal T1, respectively, with contrast indicating a relatively regular mass of $4 \times 5 \times 5 \mathrm{~cm}$ in the left base of the middle cranial fossa. (B and D) Edema surrounding the tumor, midline displacement and compressed lateral ventricle were identified. MRI, magnetic resonance imaging.

A

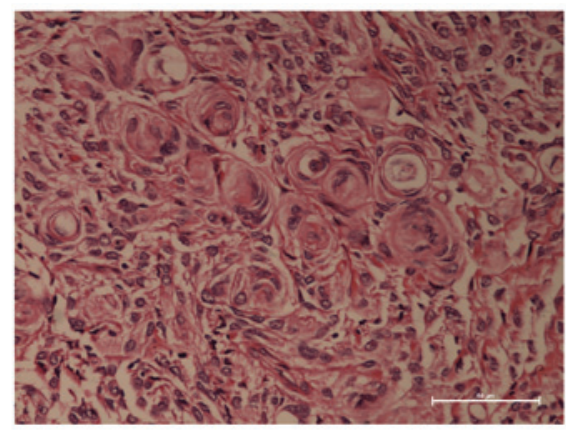

B

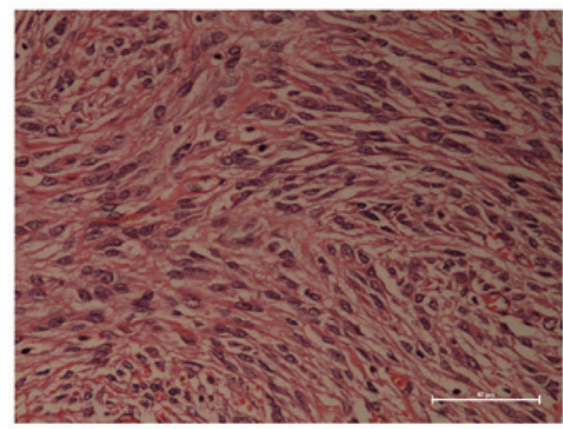

Figure 2. Pathological examination demonstrated characteristics of (A) cellular whorls and (B) significant fibroblastic collagen deposition, which indicate a transitional meningioma. (A and B) Hematoxylin and eosin staining; magnification, x200.

A

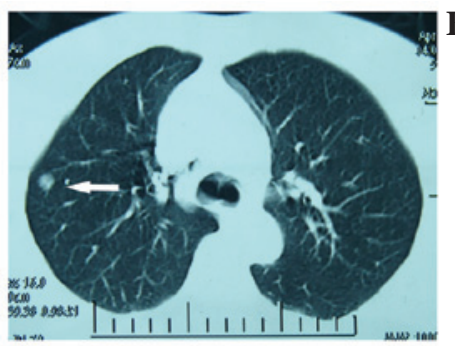

D
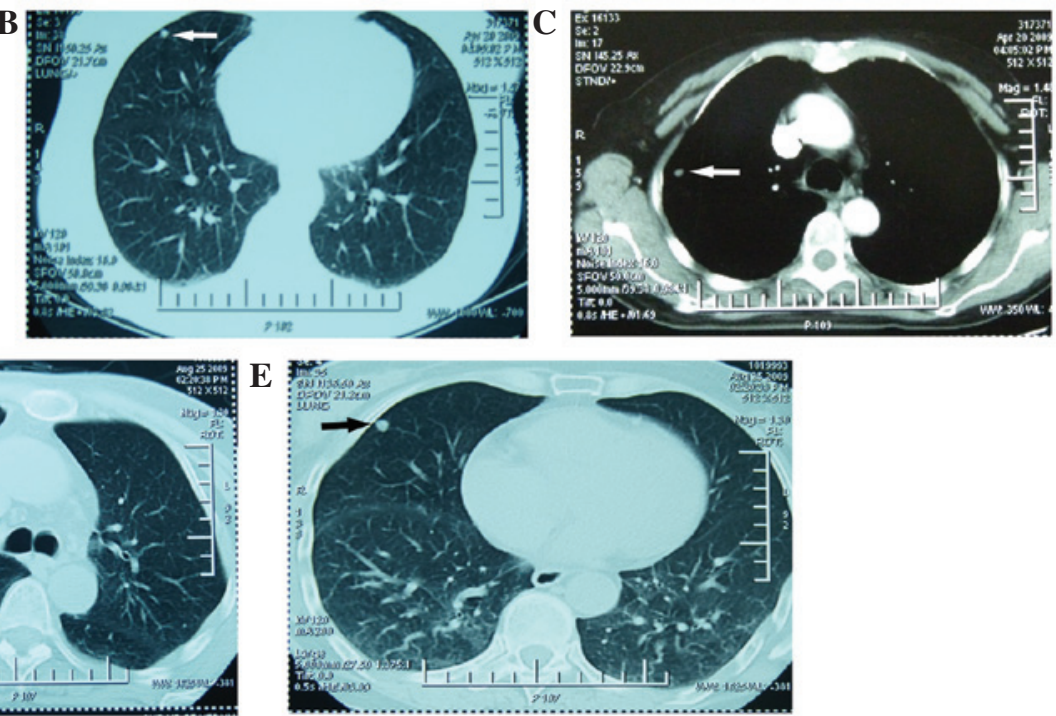

Figure 3. CT scans. (A-C) Well-circumscribed pulmonary nodules were identified in the superior lobe of the right lung (white arrows); (D) postoperative chest CT scan; and (E) follow-up CT was performed 4 months after surgery and revealed an enlarged mass located in the anterior segment (black arrow). CT, computed tomography. 

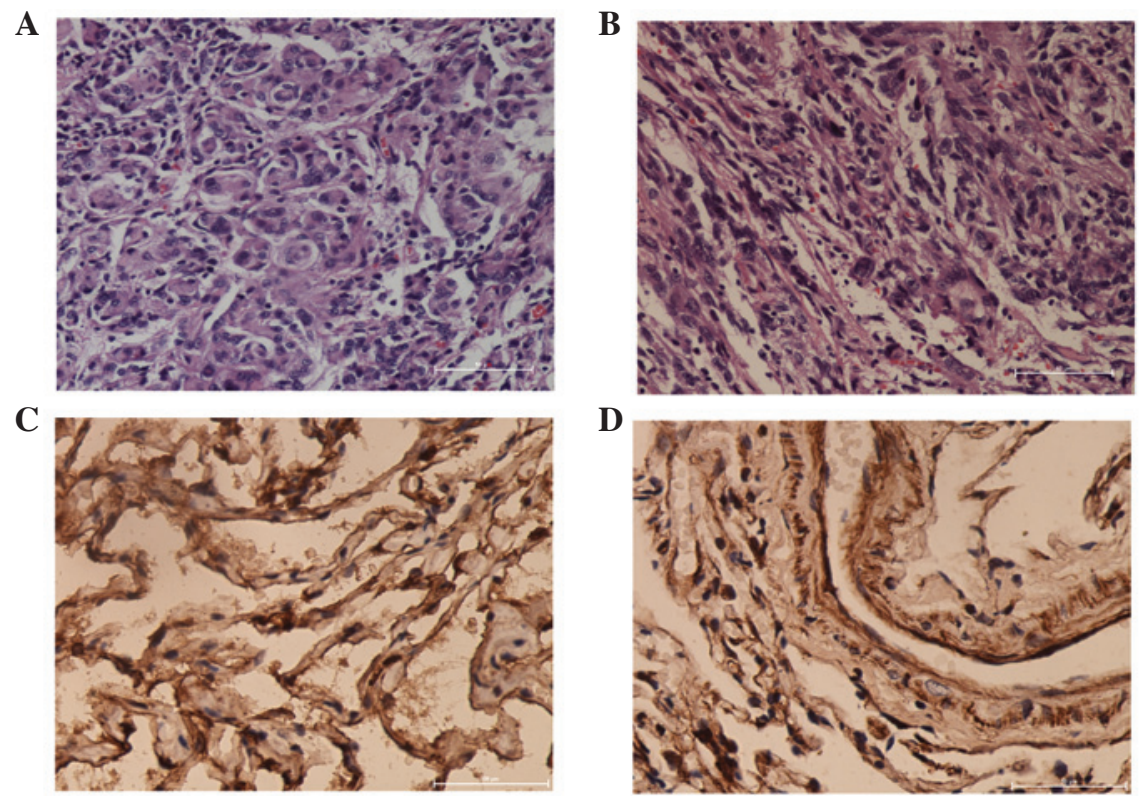

Figure 4. (A and B) Pathological examination of lung specimens indicated transitional meningioma features, similar to those of the intracranial tumor Hematoxylin and eosin staining; magnification, x200. Histological examination revealed that the tumor was positive for (C) epithelial membrane antigen and (D) vimentin, which proved the pulmonary lesions to be metastatic meningioma; magnification, $\mathrm{x} 200$.

Eighteen months following the second surgery, the patient presented with a history of dizziness for 2 months, and MRI examination revealed another tumor recurrence at the original site. A pre-operative chest $\mathrm{CT}$ scan revealed multiple pulmonary nodules in the superior lobe of the right lung (Fig. 3). Gamma Knife (dose of total, DT: $12 \mathrm{~Gy} / 50 \%, 8 \mathrm{mmx} 11$ ) was used to remove the intracranial tumor; however, a postoperative MRI indicated that the tumor had not been completely removed. In order to clarify the nature of the lesions in the right lung, wedge resection of the superior lobe of the right lung was performed 7 days later, to remove the nodules located in the junction of the tip and posterior section under direct vision of a thoracoscope. The pathological report indicated transitional meningioma features, similar to those of the intracranial tumor. Histological examination revealed that the tumor was positive for vimentin and epithelial membrane antigen (Fig. 4). The nodules in the anterior segment were placed under observation.

The patient recovered uneventfully, and was discharged from hospital 10 days post surgery. The intracranial and pulmonary residual tumors were kept under follow-up observation. Compared with the previous image, it appeared that the tumor was enlarged as indicated in the follow-up CT/MRI scans (Fig. 3E). In view of no notable clinical symptoms at that time, the patient's family refused further surgical treatment to remove the residual mass.

To date, the patient remains in a relatively stable condition, except for Broca's aphasia. Regular clinical follow-ups for the patient will continue.

\section{Discussion}

Meningiomas are slow growing tumors, which are generally considered to be benign. Meningiomas account for $15-20 \%$ of all intracranial tumors, and are twice as likely to occur in females $(1,30)$. Ectopic meningiomas are particularly rare and the majority originate from embryonic nests of arachnoid cells, which were trapped in ectopic locations during development. Hoye et al (31) classified these extracranial meningiomas into four groups, one of which is extracranial metastases of meningiomas.

Meningiomas rarely metastasize; however, cases of extracranial metastatic meningioma have previously been reported (3,4,6-9). According to current statistics, the lung is the most common site of metastasis (9). In addition, other sites, including the liver, lymph node, bone and pleura, may also occasionally exhibit metastases $(2,10,32)$. Pulmonary metastases rarely induce symptoms in the patient. They typically present as single or multiple round, non-calcified parenchymal nodules of varying size, and multiple deposits are identified in $50 \%$ of cases (8). The mean interval between diagnosis of the primary tumor and discovery of the first metastases is $\sim 6$ years (8), although the longest interval documented was 24 years (33). In addition, metastases are frequently discovered following recurrence of the primary lesion.

However, the mechanisms underlying metastasis remain to be elucidated. Hematogenous metastasis may be the most likely mechanism for extracranial spread. Figueroa et al (10) revealed that in $75 \%$ of patients reported to have had metastasizing meningioma, metastases were associated with previous surgery on the primary lesion or invasion of the sinuses. A second route underlying the development of metastasis may be via the cerebrospinal fluid pathway (12). It was hypothesized that surgical interference may release the tumor from its cohesive state into the bloodstream or cerebrospinal fluid. However, despite the relatively large number of surgical resections performed, metastasis remains a rare event (4).

The World Health Organization classification of tumors of the nervous system details 15 histopathological variants of meningioma (26). Extracranial metastases from meningiomas are considered to be a significant indicator of malignancy, and 
have been found to arise in 11-23\% of patients with anaplastic meningiomas $(2,11,34,35)$. High proliferative potential, as indicated by mitotic rate, bromodeoxyuridine labeling index, proliferating cell nuclear antigen labeling index or MIB-1 monoclonal antibody staining of $\mathrm{Ki}-67$ nuclear protein, has been suggested to enhance the risk of metastases (36). However, the clinical behavior of meningioma does not always correlate with histological features. A higher rate of cellular proliferation is not a requirement for the development of extracranial metastasis, and individual meningiomas of any histological subtype are able to metastasize $(7,10,11,37)$.

In addition, a review of the literature suggested that there may be multiple risk factors for metastasis, including previous craniotomy venous sinus invasion, local recurrence, papillary morphology and histological malignancy (13).

In the present case, the time between diagnosis of the primary tumor and detection of right lung metastasis was $\sim 2$ years and 9 months, markedly shorter than the average time. Prior to detection of the distant metastasis, multiple craniotomies, radiotherapy and Gamma Knife treatments were performed in the present case. The patient exhibited no clinical symptoms for a significant time-period prior to the detection of multiple pulmonary nodules in the superior lobe of the right lung. The present case highlights the necessity for clinicians to perform comprehensive examinations for patients with a history of recurrent intracranial meningioma in order to detect distant metastases as early as possible, even though the tumors are benign. Although there was no clear evidence of spread through the hematogenous route or cerebrospinal fluid in the present case, elucidation of the mechanisms underlying hematogenous spread remains a priority.

\section{Acknowledgements}

This study was funded by the Natioanal Natural Science Foundation of China (grant no. 61271367).

\section{References}

1. Rachlin JR and Rosenblum ML: Etiology and biology of meningiomas. In: Meningiomas. Al-Mefty O (ed). Raven Press, New York, pp27-35, 1991.

2. Pramesh CS, Saklani AP, Pantvaidya GH, Heroor AA, Naresh KN, Sharma S and Deshpande RK: Benign metastasizing meningioma. Jpn J Clin Oncol 32: 86-88, 2003.

3. Kaminski JM, Movsas B, King E, Yang C, Kronz JD, Alli PM, Williams J and Brem H: Metastatic meningioma to the lung with multiple pleural metastases. Am J Clin Oncol 24: 579-582, 2001.

4. Fulkerson DH, Horner TG and Hattab EM: Histologically benign intraventricular meningioma with concurrent pulmonary metastasis: Case report and review of the literature. Clin Neurol Neurosurg 110: 416-419, 2008.

5. Rawat B, Franchetto AA and Elavathil J: Extracranial metastases of meningioma. Neuroradiology 37: 38-41, 1995.

6. Nabeya Y, Okazaki Y, Watanabe Y, Tohnosu N, Yamazaki M, Matsuda M, Iizuka H, Akutsu N, Kono T, Sato $\mathrm{H}$ and Kubosawa H: Metastatic malignant meningioma of the liver with hypoglycemia: Report of a case. Surg Today 28: 953-958, 1998.

7. Kros JM, Cella F, Bakker SL, Paz Y, Geuze D and Egeler RM: Papillary meningioma with pleural metastasis: Case report and literature review. Acta Neurol Scand 102: 200-202, 2000.

8. Kovoor JM, Jayakumar PN, Srikanth SG, Indiria B and Devi MG: Solitary pulmonary metastasis from intracranial meningiothelial meningioma. Australas Radiol 46: 65-68, 2002.

9. Erman T,Hanta I,Haciyakupoğlu S, et al: Huge bilateral pulmonary and pleural metastasis from intracranial meningioma: A case report and review of the literature. J Neurooncol 74: 179-181, 2005.
10. Figueroa BE, Quint DJ, Mckeever PE and Chandler WF: Extracranial metastatic meningioma. Br J Radiol 72: 513-516, 1999.

11. Drummond KJ, Bittar RG and Fearnside MR: Metastatic atypical meningioma: Case report and review of the literature. J Clin Neurosci 7: 69-72, 2000.

12. Chamberlain MC and Glantz MJ: Cerebrospinal fluid-disseminated meningioma. Cancer 103: 1427-1430, 2005.

13. Adlakha A, Rao K, Adlakha H, et al: Meningioma metastatic to the lung. Mayo Clin Proc 74: 1129-1133, 1999.

14. Tao CY, Wang JJ, Li H and You C: Malignant intraventricular meningioma with craniospinal dissemination and concurrent pulmonary metastasis. World J Surg Oncol 12: 238, 2014.

15. Scognamiglio G, D'Antonio A, Rossi G, et al: CD90 expression in atypical meningiomas and meningioma metastasis. Am J Clin Pathol 141: 841-849, 2014.

16. Rico-Cotelo M, Prieto-González Á, Reyes-Santías RM, et al: Cervical lymphatic metastasis of an intracranial meningioma. Rev Neurol 56: 225-228, 2013 (In Spanish).

17. Celenk F, Erkilic S, Durucu C, Baysal E and Kanlikama M: Late metastasis of an intracranial meningioma to the hard palate. J Craniofac Surg 23: 1912-1914, 2012.

18. Wang Z, Kong M, Li J, et al: Intraspinal rhabdoid meningioma metastasis to the liver. J Clin Neurosci 18: 714-716, 2011.

19. Rampurwala M,Pazooki M and Schauer P: Delayed hepatic metastasis from a benign fibroblastic meningioma thirty-one years after surgical resection of the intracranial tumor. J Clin Oncol 29: e214-e215, 2011.

20. Moir JA, Haugk B and French JJ: Hepatic metastasis via a ventriculo-peritoneal shunt from an intracranial meningioma: Case report and review of the literature. Case Rep Gastroenterol 4: 267-272, 2010.

21. Fulkerson DH, Horner TG and Hattab EM: Histologically benign intraventricular meningioma with concurrent pulmonary metastasis: Case report and review of the literature. Clin Neurol Neurosurg 110: 416-419, 2008.

22. Akai T, Shiraga S, Iizuka H, et al: Recurrent meningioma with metastasis to the skin incision - case report. Neurol Med Chir (Tokyo) 44: 600-602, 2004

23. Williamson BE, Stanton CA and Levine EA: Chest wall metastasis from recurrent meningioma. Am Surg 67: 966-968, 2001.

24. Sironi M, Caneva A, Pasquinelli G, et al: Aspiration biopsy cytology of pleuro-pulmonary metastasis from a recurrent meningioma. Oncol Rep 4: 1165-1167, 1997.

25. Tworek JA, Mikhail AA and Blaivas M: Meningioma: Local recurrence and pulmonary metastasis diagnosed by fine needle aspiration. Acta Cytol 41: 946-947, 1997.

26. Simpson D: The recurrence of intracranial meningiomas after surgical treatment. J Neurol Neurosurg Psychiatry 20: 22-39, 1957.

27. Louis DN, Ohgaki H and Wiestler OD: The 2007 WHO classification of tumors of the central nervous system. Acta Neuropathologica 114: 97-109, 2007.

28. Shin MS, Holman WL, Herrera GA and Ho KJ: Extensive pulmonary metastasis of an intracranial meningioma with repeated recurrence: Radiographic and pathologic features. South Med J 89: 313-318, 1996.

29. Enam SA, Abdulrauf S, Mehta B, Malik GM and Mahmood A: Metastasis in meningioma. Acta Neurochir (Wien) 138: 1172-1178, 1996.

30. Demonte F and Al-Mefty O: Meningiomas. In: Brain Tumors. Kaye AH and Laws ER Jr (eds). Churchill Livingstone, New York, pp675-704, 1995.

31. Hoye SJ, Hoar CS Jr and Murray JE: Extracranial meningiomas presenting as tumor of the neck. Am J Surg 100: 486-489, 1960.

32. Karasick JL and Mullan SF: A survey of metastatic meningiomas. J Neurosurg 40: 206-212, 1974.

33. Stoller JK, Kavuru M, Mehta AC, Weinstein CE, Estes ML and Gephardt GN: Intracranial meningioma metastatic to the lung. Cleve Clin J Med 54: 521-527, 1987.

34. Evans JJ, Lee JH, Suh J and Golubic M: Neurosurgery: Principles and Practice. Moore AJ and Newell DW (eds). Springer-Verlag, London, pp205-234, 2005.

35. Kabus D, Sidhu GS, Wieczorek RL and Choi HS: Metastatic meningioma. Hemangiopericytoma or angioblastic meningioma? Am J Surg Pathol 17: 1144-1150, 1993.

36. Ohta M, Iwaki T, Kitamoto T, Takeshita I, Tateishi $\mathrm{J}$ and Fukui M: MIB1 staining index and scoring of histologic features in meningioma. Indicators for the prediction of biologic potential and postoperative management. Cancer 74: 3176-3189, 1994.

37. Hishima T, Fukayama M, Funata N, Mochizuki M, Hayashi Y, Koike $\mathrm{M}$ and Ikeda T: Intracranial meningioma masquerading as a primary pleuropulmonary tumor. Pathol Int 45: 617-621, 1995. 